\section{AB0579 INSTRUMENTS FOR SCREENING PSORIATIC ARTHRITIS AMONG PATIENTS WITH PSORIASIS: A SYSTEMATIC LITERATURE REVIEW}

G. Can ${ }^{1}$, G. Ayan ${ }^{2}$, A. Ozdede ${ }^{3}$, M. Bektaş ${ }^{4}$, N. Akdogan ${ }^{5}$, B. YaliciArmagan $^{5}$, E. Oksum Solak ${ }^{6}$, S. Yazici ${ }^{7}$, U. Kalyoncu ${ }^{2}$, E. Ozsoy Adisen ${ }^{8}$, N. Atakan ${ }^{5}$, E. Bulbul Baskan ${ }^{7}$, M. Borlu ${ }^{6}$, B. Engin ${ }^{9}$, V. Hamuryudan ${ }^{3}$, M. Inanc ${ }^{4}$, S. Kiraz ${ }^{2}$, F. Onen ${ }^{1}$, S. Ugurlu ${ }^{3}$, S. Yayli ${ }^{10}$, G. Hatemi ${ }^{3} .{ }^{1}$ Dokuz Eylul University, Medical Faculty, Department of Internal Medicine Division of Rheumatology, Izmir, Turkey; ${ }^{2}$ Hacettepe University Medical Faculty, Department of Internal Medicine Division of Rheumatology, Ankara, Turkey; ${ }^{3}$ Istanbul University-Cerrahpasa, Cerrahpasa Medical Faculty, Department of Internal Medicine Division of Rheumatology, ISTANBUL, Turkey; ${ }^{4}$ stanbul University Istanbul Medical Faculty, Department of Internal Medicine Division of Rheumatology, ISTANBUL, Turkey; ${ }^{5}$ Hacettepe University Medical Faculty, Department of Dermatology, ANKARA, Turkey; ${ }^{6}$ Erciyes University Medical Faculty, Department of Dermatology, Kayseri, Turkey; ${ }^{7}$ Uludag University Medical Faculty, Department of Dermatology, Bursa, Turkey; ${ }^{8}$ Gazi University Gazi Medical Faculty, Department of Dermatology, ANKARA, Turkey; ${ }^{9}$ Istanbul University-Cerrahpasa, Cerrahpasa Medical Faculty, Department of Dermatology, ISTANBUL, Turkey; ${ }^{10}$ Karadeniz Technical University, Medical Faculty, Department of Dermatology, Trabzon, Turkey

Background: Timely diagnosis is essential for the optimal management of psoriatic arthritis (PsA). Several instruments have been developed for screening PsA among patients with psoriasis. However, a delay in diagnosis is still frequently reported, possibly due to the lack of a wide use of these instruments.

Objectives: We aimed to identify and compare the reported performance of these instruments with special emphasis on the PsA phenotypes.

Methods: We conducted a systematic literature search on PubMed until 15 August 2020 using the keyword 'psoriatic arthritis'. Two independent reviewers identified all studies published in English, that report on the validation, psychometric evaluation or use of an instrument for screening PsA. Any disagreements were resolved by the third investigator. Data on sensitivity, specificity, positive (PPV) and negative (NPV) predictive values were extracted or calculated for each instrument. Additionally, instruments were assessed for their performance in patients with different disease phenotypes.

Results: A total of 10754 references were screened, and 42 were identified that reported on 15 different screening instruments. Psoriatic Arthritis Screening and Evaluation (PASE), Psoriasis Epidemiology Screening Tool (PEST), Early Arthritis for Psoriatic Patients questionnaire (EARP) were the most commonly used instruments. There was important variability across studies regarding the sensitivity, specificity, PPV and NPV of these instruments based on the cut-offs for positivity, setting, patient population and disease phenotypes (Table 1). Specificity was higher when patients with a previous diagnosis of other rheumatic diseases were excluded. Lower sensitivity was reported among patients with shorter disease duration and when patients with a prior diagnosis of PsA were excluded from the study, whereas higher sensitivity was reported among patients with prior NSAID use. Screening tools showed differences in sensitivity in different domains (Figure 1).
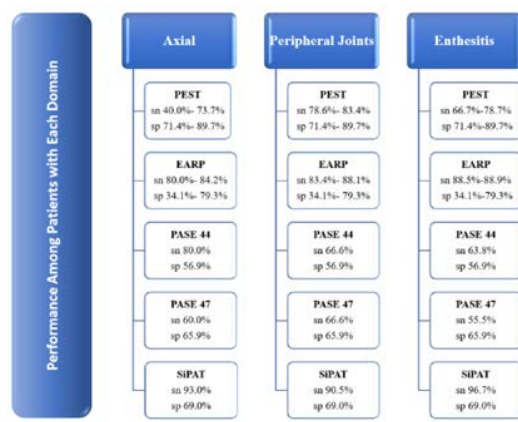

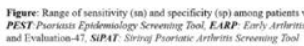

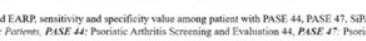

Figure 1. Performance Among Patients with Each Domain

Conclusion: This systematic literature review revealed wide variability in the diagnostic estimates of currently available questionnaire-based screening instruments for identifying PsA among psoriasis patients, depending on study populations and disease phenotypes. There is an unmet need for a screening instrument with a better performance in all disease domains.
Table 1. Diagnostic estimates of screening tools in different studies

\begin{tabular}{llllll}
\hline Instrument & $\begin{array}{l}\text { Number of } \\
\text { studies }\end{array}$ & $\begin{array}{l}\text { Sensitivity } \\
\%\end{array}$ & $\begin{array}{l}\text { Specificity } \\
\%\end{array}$ & $\begin{array}{l}\text { PPV } \\
\%\end{array}$ & $\begin{array}{l}\text { NPV } \\
\%\end{array}$ \\
\hline PASE & 18 & $24-91$ & $38-95$ & $18-88$ & $13-96$ \\
PEST & 11 & $40-85$ & $37.2-98.6$ & $23-96$ & $47.1-99.3$ \\
EARP & 9 & $41-97.2$ & $34-97.2$ & $14-93.3$ & $57.5-100$ \\
TOPAS & 6 & $41-89.1$ & $29.7-90$ & $25.7-91.8$ & $68-81.6$ \\
TOPAS-II & 4 & $44-95.8$ & $80.5-98$ & $63.4-95.8$ & $91-98$ \\
PSA-Disk questionnaire & 1 & 87.2 & 46.4 & 58.6 & 78.5 \\
CONTEST & 2 & $70-76.5$ & $56.5-91$ & $16-89$ & $68-95$ \\
STRIPP & 1 & 91.5 & 93.3 & 79.6 & 97.5 \\
SiPAS & 1 & 79 & 87 & 73 & 90 \\
PASQ & 2 & $67-92.7$ & $64-81.8$ & 43 & 83 \\
GEPARD & 2 & 77 & 70 & 66 & 80 \\
Swedish- Psoriasis & 1 & 63 & 72 & 45 & 85 \\
$\quad$ Assessment Questionnaire & 1 & 60 & 62 & 26 & 87.5 \\
PAQ & 1 & 69 & 69 & 91 & 69 \\
SiPAT & 1 & 86.9 & 71.3 & 53 & 93.6 \\
A novel, short, and simple & 1 & & & & \\
$\quad$ screening questionnaire & & & & &
\end{tabular}

PASE: Psoriatic Arthritis Screening and Evaluation, PEST: Psoriasis Epidemiology Screening Tool, EARP: Early Arthritis for Psoriatic Patients questionnaire, TOPAS: Toronto Psoriatic Arthritis Screening Questionnaire, STRIPP: Screening Tool for Rheumatologic Investigation,SIPAS: Simple Psoriatic Arthritis Screening questionnaire, PASQ: Psoriasis and Arthritis Screening Questionnaire, GEPARD: German Psoriatic Arthritis Diagnostic Questionnaire, PAQ: Psoriatic and Arthritic Questionnaire, SiPAT: Siriraj Psoriatic Arthritis Screening Tool

Disclosure of Interests: None declared.

DOI: 10.1136/annrheumdis-2021-eular.3275

\section{$\mathrm{AB} 0580$ \\ EFFECT OF FATIGUE ON DISEASE ACTIVITY AND PATIENT-REPORTED OUTCOMES (PROS) IN PATIENTS (PTS) WITH PSORIATIC ARTHRITIS (PSA)}

L. Vorobyova ${ }^{1}$, T. Korotaeva ${ }^{1}$, E. Loginova ${ }^{1}$, Y. Korsakova ${ }^{1}$, E. Gubar ${ }^{1}$,

P. Tremaskina ${ }^{1}$. $V$. A. Nasonova Research Institute of Rheumatology,

Department of Spondyloarthritis and Psoriatic Arthritis, Moscow, Russian Federation

Background: Fatigue is an important symptom in patients (pts) with psoriatic arthritis (PsA) and was recently added to the core domain set for PsA randomized controlled trials (RCTs) due to the impact that it has on a PROs.

Objectives: to study the influence of fatigue on disease activity and PROs in pts with PsA.

Methods: 187 (M/F=97 (50.2\%)/90(48.8\%) PsA pts fulfilling the CASPAR criteria were included. Mean age $45.6+11.7$ years (yrs), median (Me) PsA duration 88 [16;421] mo. $67,8 \%$ of pts $67,8 \%$ were treat biological DMARDs, $32,1 \%$ of pts was monotherapy conventional synthetic disease-modifying antirheumatic drugs (csDMARDs, mostly Methotrexate). On visit PsA activity was evaluated by Tender Joint Count (TJC68), Swollen Joint Count (SJC66), DAPSA. PROs were measured by PtGA VAS, PtPain VAS, BASDAI, HAQ, EQ-5D, PsAID12, WPAI. Fatigue symptoms were assessed by the Functional Assessment of Chronic Illness Therapy-Fatigue (FACIT-Fatigue) scale. Higher PsAID12 scores are considered to be worse and correspond to poorer PsA-specific health-related quality of life. $\mathrm{M} \pm \mathrm{SD}$, Me [Q25; Q75], \%, t-test, Manna-Whitney tests were performed. Al $\mathrm{p}<0.05$ were considered to indicate statistical significance.

Table 1. The effect fatigue on disease activity and PROs

\begin{tabular}{|c|c|c|c|}
\hline Parameters, $\mathrm{M} \pm \mathrm{SD}$ & $\begin{array}{l}\text { No fatigue } \\
n=99\end{array}$ & $\begin{array}{l}\text { Fatigue } \\
n=68\end{array}$ & $\begin{array}{l}\text { Severe } \\
\text { fatigue } \\
n=20\end{array}$ \\
\hline TJC68 & $9.51 \pm 7.86$ & $14.5 \pm 9.89^{*}$ & $19.05 \pm 8.15^{\star *}$ \\
\hline SJC66 & $7.66 \pm 5.5$ & $11.5 \pm 8.2^{*}$ & $13.4 \pm 6.9^{\star \star}$ \\
\hline BASDAI & $2.6 \pm 1.85$ & $5.8 \pm 2.47^{*}$ & $7.2 \pm 2.0^{\star \star}$ \\
\hline DAPSA & $21.05 \pm 21.03$ & $39.9 \pm 21.13^{*}$ & $47.9 \pm 15.6^{\star \star}$ \\
\hline $\mathrm{CRP}(\mathrm{mg} / \mathrm{l})$ & $12.6 \pm 9.4$ & $30.0 \pm 25.4^{\star}$ & $20.9 \pm 11.6^{\star \star}$ \\
\hline PtPain, VAS, mm & $33 \pm 24.7$ & $59 \pm 25.2^{*}$ & $66.8 \pm 18.1^{\star \star}$ \\
\hline PtGA, VAS, mm & $30.7 \pm 23.8$ & $54.0 \pm 22.2^{*}$ & $64.7 \pm 16.2^{* *}$ \\
\hline $\operatorname{ESR}(\mathrm{mm} / \mathrm{h})$ & $15.19 \pm 11.7$ & $30.1 \pm 23.7^{\star}$ & $30.1 \pm 20.8^{\star \star}$ \\
\hline Total PsAID12 score (over past week, 0- 10) & $1.87 \pm 1.76$ & $5.41 \pm 2.17^{*}$ & $6.5 \pm 1.93^{\star \star}$ \\
\hline $\mathrm{HAQ}$ & $0.72 \pm 0.59$ & $1.37 \pm 0.84^{\star}$ & $2.04 \pm 0.86^{\star *}$ \\
\hline EQ-5D TTO & $0.75 \pm 0.17$ & $0.46 \pm 0.16^{*}$ & $0.51 \pm 0.15^{\star \star}$ \\
\hline Work time missed (absenteeism) & $0.08 \pm 0.26$ & $0.11 \pm 0.25$ & $0.31 \pm 0.15^{\star \star}$ \\
\hline Impairment while working (presenteeism) & $0.085 \pm 0.15$ & $0.258 \pm 0.256^{*}$ & $0.23 \pm 0.13^{\star \star}$ \\
\hline $\begin{array}{l}\text { Overall work productivity impairment (absenteeism } \\
\text { + presenteeism) }\end{array}$ & $0.15 \pm 0.26$ & $0.35 \pm 0.34^{\star}$ & $0.37 \pm 0.26^{\star \star}$ \\
\hline Daily activity impairment & $0.22 \pm 0.23$ & $0.59 \pm 0.25^{\star}$ & $0.73 \pm 0.22^{\star *}$ \\
\hline
\end{tabular}

${ }^{*} \mathrm{p}<0.001$ - differences from "No fatigue" to "Fatigue"; ${ }^{* *} \mathrm{p}<0.0001$ - differences from "No fatigue" to "Severe Fatigue"; 